\title{
Receptor Pair for Schizophrenia
}

$\mathrm{T}$ hree different neurotransmitters, dopamine, serotonin, and glutamate, have each spawned hypotheses about the neurochemical alterations in schizophrenia. The receptors for these neurotransmitters, as well as for many therapeutic drugs, are members of the largest class of signaling molecules in the human genome, the G-protein coupled receptors (GPCRs). The recent identification of a molecular complex in the brain made up of a pair of GPCRs, one for glutamate and another for serotonin, may provide insight into the neurochemistry of schizophrenia and the mechanism of action of antipsychotic medications commonly used in pediatric patients (1).

The discovery of the structural and functional relationship of these receptors evolved from work on the mechanism of action of hallucinogenic chemicals that are related to serotonin. Natural hallucinogens, such as mescaline or psilocybin, and synthetic hallucinogens, such as lysergic acid diethylamide, cause their neuropsychological effects by activating the serotonin $2 \mathrm{~A}$ receptor, a brain GPCR. Because the effects of hallucinogens have only some similarities to the perceptual and affect disturbances experienced in schizophrenia, the usefulness of studying hallucinogens as a model for schizophrenia has been debated. Supporting the relevance of studying hallucinogens to understand schizophrenia, however, is the observation that the serotonin $2 \mathrm{~A}$ receptor also interacts with atypical antipsychotic medications used to treat schizophrenia, such as risperidone and clozapine.

Surprisingly, the serotonin $2 \mathrm{~A}$ receptor was discovered to form a functional molecular complex with a different GPCR, the metabotropic glutamate 2 receptor. Interestingly, the brains of untreated patients with schizophrenia showed increased levels of the serotonin receptor and decreased levels of the glutamate receptor. The recent success of a phase II clinical trial for a member of a new class of antipsychotic drugs that binds to and activates the metabotropic glutamate 2 receptor also suggests that this complex may play a role in the altered thinking processes of schizophrenia (2).

The new complex includes the serotonin receptor that binds hallucinogens and is blocked by atypical antipsychotic medications, as well as the glutamate receptor that binds glutamate antipsychotic drugs under development. This suggests that these two different classes of schizophrenia drugs may be acting at different components of the same molecular complex. Supporting this notion are several observations. Lysergic acid diethylamide and other hallucinogens cause a particular pattern of neuronal signaling when acting at this complex. Further, the hallucinogen response is suppressed by drugs that activate the glutamate receptor component. Perhaps the changes in the two receptors found in the brains of untreated patients with schizophrenia alter the integration of glutamate and serotonin signaling in a way that predisposes to psychosis.

The first class of drugs developed for schizophrenia, which includes antipsychotics like chlorpromazine and haloperidol, are believed to work via a different GPCR target, the dopamine D2 receptor. At this time, the relationship of dopamine signaling to the serotoninglutamate receptor complex is unknown. Nonetheless, the discovery of the serotonin-glutamate receptor pair suggests that the mechanisms for the perceptual disturbances caused by hallucinogenic drugs of abuse, for the disordered thought processes in schizophrenia and for the actions of two important classes of antipsychotic medications may be related. The existence of this complex could help integrate hypotheses about the contributions of serotonin signaling and of glutamate signaling to the symptoms of schizophrenia and may facilitate the development of drugs to treat psychosis. - Stuart C. Sealfon and Javier González-Maeso

\section{REFERENCES}

1. Gonzalez-Maeso J, Ang RL, Yuen T, Chan P, Weisstaub NV, Lopez-Gimenez JF, Zhou M, Okawa Y, Callado LF, Milligan G, Gingrich JA, Filizola M, Meana JJ, Sealfon SC 2008 Identification of a serotonin/glutamate receptor complex implicated in psychosis. Nature 452:93-97

2. Patil ST, Zhang L, Martenyi F, Lowe SL, Jackson KA, Andreev BV, Avedisova AS, Bardenstein LM, Gurovich IY, Morozova MA, Mosolov SN, Neznanov NG, Reznik AM, Smulevich AB, Tochilov VA, Johnson BG, Monn JA, Schoepp DD 2007 Activation of mGlu2/3 receptors as a new approach to treat schizophrenia: a randomized phase 2 clinical trial. Nat Med 13:1102-1107 